Scientific Review - Engineering and Environmental Sciences (2017), 26 (4), 528-537

Sci. Rev. Eng. Env. Sci. (2017), 26 (4)

Przegląd Naukowy - Inżynieria i Kształtowanie Środowiska (2017), 26 (4), 528-537

Prz. Nauk. Inż. Kszt. Środ. (2017), 26 (4)

http://iks.pn.sggw.pl

DOI 10.22630/PNIKS.2017.26.4.50

\author{
Alexandr A.TRESCHEV ${ }^{\mathbf{1}}$, Maria V. SPASSKAYA ${ }^{\mathbf{1}}$, \\ Michael B. SHERESHEVSKY ${ }^{1}$, Alexander A. BOBRISHEV ${ }^{2}$, \\ Lenar N. SHAFIGULLIN ${ }^{2}$ \\ ${ }^{1}$ Department of Engineering, Constructional Materials and Structures, \\ Tula State University \\ ${ }^{2}$ Department of Materials, Technologies and Quality \\ Kazan (Volga region) Federal University
}

\title{
The influence of temperature differences for the analysis of thin orthotropic cylindrical shell
}

Key words: cylindrical shell, temperature differential, orthotropy

\section{Introduction}

Nowadays material science is constantly evolving, new materials with complex properties appear. These and many classic constructional materials exhibit different resistant and structural anisotropic properties. Different resistant is a material whose deformational and structural properties depend on type of stress state. Anisotropic is a material whose physical and mechanical properties differ depending on direction. Classic theories of structural mechanics and solid mechanics do not provide sufficient accuracy of calculations of stress-strain state characteristics of materials with complex properties, with critical struc- tures becoming more common in chemical, constructional, machine and aviation building industries at the present time. Therefore, it is necessary to create dependable theories that agree with experimental data.

Besides, temperature effect on stress-strain state of structures made of materials with complex properties needs more consideration. Works in the field of thermo-elasticity of anisotropic different resistant materials are quite chaotic, and since new structures work under greater temperature gradient it is of utmost importance to systematically research thermo-mechanical problems of anisotropic different resistant materials within the framework of current structural mechanics, solid mechanic and its practical implementation.

The materials in question have large modules of elasticity and other me- 
chanical strength characteristics, therefore they are often used in thin structures such as plates and shells. Thin shells fall into category of spatial structures and are used in constructions with big open spaces. The thin shell has the form of curved surface that with it having minimal width, mass and material consumption possesses quite big bearing capability due to its curved shape.

From now forth we are considering a special case - a circular cylindrical shell. In practical cases, it is often symmetrical about axis of cylinder forces that effect the shell. Cylindrical boilers that suffer vapor pressure, vertical cisterns and round pipes that suffer water pressure - are all examples of such problems. It is obvious that all structures mentioned above in addition to evenly distributed load suffer temperature action.

Effect of coupling of deformation and temperature fields for many different resistant anisotropic materials is quite small. Therefore, in case of typical heat transfer in non-uniformly heated body by means of thermal action of environment, influence of body's deformations on its temperature distribution is a value to be thrown away. This allows us to analyze a temperature field that correspond to specific conditions of heat transfer

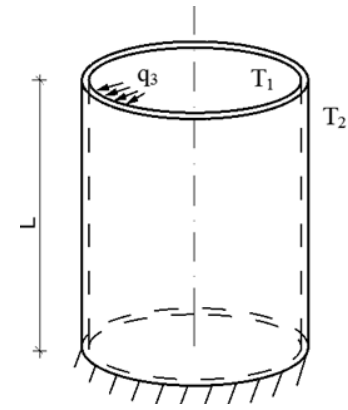

FIGURE 1. Initial scheme independently from stress-strain state of the body. That is why it is reasonable to consider an uncoupled problem of thermo-elasticity.

Therefore, it may be concluded that consideration of anisotropy and different resistance as well as influence of temperature action on stress-strain state is a relevant objective in terms of both science and application.

\section{Problem definition}

Circular cylindrical shell made of orthotropic different resistant material is in question (Spasskaya, 2013). Main axes of orthotropy coincide with axes of main stresses. Geometrical characteristics of the shell: height $-L$, depth $-h$, radius of middle surface $-R$. With $\beta_{1}=0$ one end of cylindrical shell is rigidly fixed, and the other with $\beta_{1}=L$ is free with no load. The shell suffers temperature differential: the temperature of the inner $T_{1}$ and outer $T_{2}$ surfaces, as well as evenly distributed load $q_{3}$ on inner surface of the shell (inner pressure), as shown on Figure 1. Let's assume that temperature differentiation on inner and outer surfaces $\Delta T$ is a function of coordinate $\beta_{3}$. The position of any point of the shell is de-

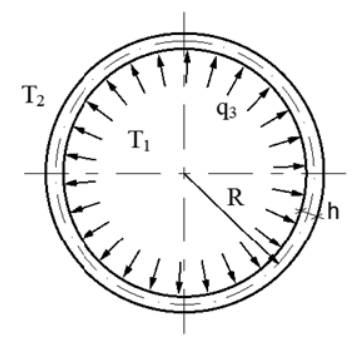


fined in Gaussian coordinate system $\beta_{1}$, $\beta_{2}, \beta_{3}$. The position of any point on the middle surface of the cylindrical shell is defined by Gaussian coordinates $\beta_{1}$ and $\beta_{2}$.

The shell is working within the framework of shallow shells theory that uses the following dependences:

a) components of deformation in middle surface (stretch and shear):

$\varepsilon_{1}=u,_{1}+0.5 \theta_{1}^{2} ; \varepsilon_{2}=v_{,}+k w+0.5 \theta_{2}^{2}$

$\gamma=v,{ }_{1}+u,,_{2}+\theta_{1} \theta_{2}$

where:

$\varepsilon_{1}, \varepsilon_{2}-$ stretching deformations;

$\gamma$ - shear;

$u, v, w$ - axial, tangential and radial displacements;

$k=1 / R-$ main curvature;

$\theta_{1}, \theta_{2}-$ rotations of middle surface normal:

$\theta_{1}=-w, \theta_{2}=-w,,_{2}+k v$

b) components of bending deformation (change of curvature and tortion):

$\chi_{1}=-w,{ }_{11} ; \chi_{2}=-w,{ }_{22} ; \tau=-w,,_{12}$

where:

$\chi_{1}, \chi_{2}$ - curvatures;

$\tau$ - torsion;

c) components of deformation in a point that lays at a distance $\beta_{3}$ from the middle surface, expressed in terms of components of tangential and bending deformations:

$$
\begin{aligned}
& e_{11}=\varepsilon_{1}+\beta_{3} \chi_{1} ; e_{22}=\varepsilon_{2}+\beta_{3} \chi_{2} \\
& \gamma_{12}=\gamma+2 \beta_{3} \tau
\end{aligned}
$$

Expressions (1)-(4) are called kinematic correlations. They are correct for theory of shallow shells within quadratic approximation with deformations being small and elastic.

Let's assume the shell is quite thin, in addition to that let's use traditional hypotheses of Kirchhoff-Love.

In order to get equations that consider temperature action, equations of mechanics have to be supplemented with corresponding temperature components.

Correlations of Treschev (2016) will play part of physical correspondences. The body is assumed orthotropic in order to specify type of anisotropy.

Considering assumed hypotheses and axes of cylindrical coordinate system coinciding with main axes of anisotropy physical dependences will look like:

$$
\begin{aligned}
& e_{11}=\left(A_{1111}+B_{1111} \alpha_{11}\right) \sigma_{11}+\left\lfloor A_{1122}+B_{1122}\left(\alpha_{11}+\alpha_{22}\right)\right] \sigma_{22}+\alpha_{1 T} \Delta T \\
& e_{22}=\left[A_{1122}+B_{1122}\left(\alpha_{11}+\alpha_{22}\right)\right] \sigma_{11}+\left(A_{2222}+B_{2222} \alpha_{22}\right) \sigma_{22}+\alpha_{2 T} \Delta T \\
& e_{12}=\left(A_{1212}+B_{1212} \sqrt{2} \alpha_{12}\right) \tau_{12}
\end{aligned}
$$

where:

$A_{k k k k}, B_{k k k k}, A_{i i j j}, B_{i i j j}, A_{i j i j}, B_{i j i j}$ - constants, that depend from modules of elasticity and Poisson's ratios;

$\alpha_{i j}=\sigma_{i j} / S-$ normalized stresses;

$S=\sqrt{\sigma_{i j} \sigma_{i j}}-$ modulus of vector of total stresses;

$\alpha_{1 T}, \alpha_{2 T},-$ coefficients of thermal linear expansion in due directions;

$\Delta T$ - temperature differentiation in any point through the thickness of the shell.

Determination of constants of resolving equations for orthotropic materials should be based on results of simple experiments: axial tension and compression along main axes of anisotropy, shear in main surfaces or axial tension or compression in directions with an angle of 
$45^{\circ}$ to corresponding main axes of anisotropy (Treschev, 2016). With constants for orthotropic body being:

$$
\begin{array}{lc}
A_{k k k k}=\left(1 / E_{k}^{+}+1 / E_{k}^{-}\right) / 2 ; & B_{k k k k}=\left(1 / E_{k}^{+}-1 / E_{k}^{-}\right) / 2 \\
A_{i j j j}=-\left(v_{i j}^{+} / E_{j}^{+}+v_{i j}^{-} / E_{j}^{-}\right) / 2 ; & B_{i j j}=-\left(v_{i j}^{+} / E_{j}^{+}-v_{i j}^{-} / E_{j}^{-}\right) / 2 \\
A_{i j i j}=\left(1 / E_{i j}^{+}+1 / E_{i j}^{-}\right)-0.25\left[\begin{array}{l}
\left(1 / E_{i}^{+}+1 / E_{j}^{+}+1 / E_{i}^{-}+1 / E_{j}^{-}\right)- \\
-2\left(v_{j i}^{+} / E_{i}^{+}+v_{j i}^{-} / E_{i}^{-}\right)
\end{array}\right] \\
B_{i j i j}=\sqrt{2}\left(1 / E_{i j}^{+}-1 / E_{i j}^{-}\right)-0.125 \cdot \sqrt{2} \cdot\left[\begin{array}{l}
\left(1 / E_{i}^{+}+1 / E_{j}^{+}-1 / E_{i}^{-}-1 / E_{j}^{-}\right)- \\
-4\left(v_{j i}^{+} / E_{i}^{+}-v_{j i}^{-} / E_{i}^{-}\right)
\end{array}\right]
\end{array}
$$

where:

$v_{i j}^{+} / E_{j}^{+}=v_{j i}^{+} / E_{i}^{+} ; v_{i j}^{-} / E_{j}^{-}=v_{j i}^{-} / E_{i}^{-} ;$

$E_{k}^{ \pm}, E_{i}^{ \pm}, E_{j}^{ \pm}-$modules of elasticity for case of strain and compression in directions along main axes of anisotropy; $v_{i j}^{ \pm}, v_{j i}^{ \pm}$- Poisson's ratios for case of strain and compression in directions along main axes of anisotropy;

$E_{i j}^{ \pm}-$modules of elasticity for case of strain and compression in directions with an angle of $45^{\circ}$ to corresponding main axes of anisotropy.

\section{Derivation of resolving equations}

In order to get the system of resolving equations, first it is necessary to reform physical dependences in form of those used in the method of elastic solutions of Ilushin (1963) and express them in terms of deformations:

$$
\begin{aligned}
& \sigma_{11}=C_{11} e_{11}+C_{12} e_{12}-R_{11}-\varphi_{1 T} \\
& \sigma_{22}=C_{12} e_{11}+C_{22} e_{12}-R_{22}-\varphi_{2 T} \\
& \tau_{12}=C_{66} e_{12}-R_{12}
\end{aligned}
$$

where:

$C_{11}=A_{2222} / \Delta ; C_{12}=-A_{1112} / \Delta ; C_{22}=$ $=\mathrm{A}_{111} / \Delta ; C_{66}=1 / A_{1112}$

$R_{11}=\left(A_{2222} T_{11}-A_{1111} T_{22}\right) / \Delta ; \mathrm{R} 22=$ $=\left(A_{1111} T_{22}-A_{1122} T_{11}\right) / \Delta$

$R_{12}=T_{12} / A_{1122} ; T_{11}=B_{1111} \alpha_{11} \sigma_{11}+$ $+B_{1122}\left(\alpha_{11}+\alpha_{22}\right) \sigma_{22}$

$T_{12}=B_{1122} \sqrt{2 \alpha_{12} \tau_{12}} ; T_{22}=B_{2222} \alpha_{22} \sigma_{22}+$ $+B_{1122}\left(\alpha_{11}+\alpha_{22}\right) \sigma_{11}$

$\varphi_{1 T}=C_{11} \alpha_{1 T} \Delta T+C_{12} \alpha_{1 T} \Delta T ; \varphi_{2 T}=$ $=C_{12} \alpha_{1 T} \Delta T+C_{22} \alpha_{2 T} \Delta T$

$\Delta=A_{1111} A_{2222}-A_{1122}^{2}$

The choice of determining equations does not affect static-geometric correlations. Therefore, main postulates of geometrically non-linear theory of anisotropic shells are true. Inner forces and moments are transformed to middle surface $\beta_{3}=0$, and considering $\beta_{3} k<<1$ equilibrium equations for shallow shells are defined by:

$$
\begin{aligned}
& N_{1,1}+S,_{2}+q_{1}=0 \\
& N_{2,,_{2}}+S,_{1}+k\left(Q_{2}+H_{, 1}\right)+q_{2}=0 \\
& M_{1,1}+H,_{2}-Q_{1}-N_{1} \theta_{1}-S \theta_{2}=0
\end{aligned}
$$


$M_{2,2}+H_{, 1}-Q_{2}-N_{2} \theta_{2}-S \theta_{1}=0$

$Q_{1,1}+Q_{2,2}+k N_{2}+q_{3}=0$

where:

$N_{k}, S$ - forces in the middle surface of the shell;

$Q_{k}$ - shear forces;

$q_{m}$ - load densities on corresponding directions;

$M_{k}, H$ - bending and rotational moments.

These characteristics can be found as following, because transition from stresses to their integral characteristics - forces and moments - does not depend on physical nature of the material:

$$
N_{k}=\int_{-h / 2}^{h / 2} \sigma_{k k} d \beta_{3} ; M_{k}=\int_{-h / 2}^{h / 2} \sigma_{k k} \beta_{3} d \beta_{3}
$$$$
Q_{k}=\int_{-h / 2}^{h / 2} \sigma_{k 3} d \beta_{3} ; H=\int_{-h / 2}^{h / 2} \sigma_{12} d \beta_{3}
$$$$
H=\int_{-h / 2}^{h / 2} \sigma_{12} \beta_{3} d \beta_{3}
$$

Equations of strain compatibility:

$$
k \chi_{1}+\chi_{1} \chi_{2}-\tau^{2}-\gamma,,_{12}+\varepsilon_{1,22}+\varepsilon_{2,11}=0
$$

The shell is suffering a load in a form of internal pressure $-q_{3}$. With the problem being symmetrical, all parameters of stress-strain state will vary depending on coordinate $\beta_{1}$. That is why dependencies (1)-(4), (6) and (8) look:

- kinematic dependencies:

$\varepsilon_{1}=u,,_{1}+0.5 \theta_{1}^{2} ; \varepsilon_{2}=k w ; \chi_{1}=-w,,_{11}$

$\mathrm{e}_{11}=\varepsilon_{1}+\beta_{3} \chi_{1} ; e_{22}=\varepsilon_{2}$
- equations of equilibrium:

$$
\begin{aligned}
& N_{1,1}=0 ; M_{1,1}-Q_{1}-N_{1} \theta_{1}=0 \\
& \mathrm{Q}_{1,1}-k N_{2}+q_{3}=0
\end{aligned}
$$

- equation of strain compatibility:

$k \chi_{1}+\varepsilon_{2,11}=0$

Let's integrate correlations (5) though-the-thickness of the shell in accordance with (7) and place the resulting dependencies for shear force in equilibrium equations (10). Besides, by using equation of strain compatibility (11), we finally derive a system of two differential equations in combined form relating to unknown degree of rotation and axial force in tangential direction:

$\left\{\begin{array}{l}\theta_{1,1}+R L_{22} N_{2,11}=-R \eta_{2,11}-R \eta_{2 T}, 11 \\ \theta_{1,11}-\frac{1}{R P_{11}} N_{2}=-\frac{1}{P_{11}} q_{3}+\frac{1}{P_{11}} J_{11,1}+\frac{1}{P_{11}} \chi_{1 T},\end{array}\right.$

where:

$L_{22}=\frac{C_{11}}{\left(C_{11} C_{22}-C_{12}^{2}\right) h}$

$P_{11}=C_{11} h^{3} / 12$

$\eta_{2}=-\frac{C_{12}}{\left(C_{11} C_{22}-C_{12}^{2}\right) \cdot h} I_{11}+L_{22} I_{22}$

$\eta_{2 T}=-\frac{C_{12}}{\left(C_{11} C_{22}-C_{12}^{2}\right) \cdot h} \varepsilon_{1 T}+L_{22} \varepsilon_{2 T}$

$I_{i j}=\int_{-h / 2}^{h / 2} R_{i j} d \beta_{3} ; \quad J_{i j}=\int_{-h / 2}^{h / 2} R_{i j} \beta_{3} d \beta_{3}$

$\varepsilon_{i T}=\int_{-h / 2}^{h / 2} \phi_{i T} d \beta_{3} ; \quad \chi_{i T}=\int_{-h / 2}^{h / 2} \phi_{i T} \beta_{3} d \beta_{3}$ 
As one end of the shell is rigidly fixed with $\beta_{1}=0$ and stretching deformations in the middle surface of the shell are equal to zero, boundary conditions with $\beta_{1}=0$ are:

$\theta_{1}=0 ; \varepsilon_{1}=0 ; \varepsilon_{2}=0 \Rightarrow N_{2}=-\frac{1}{L} \eta_{2}$

\section{Temperature component of the problem}

Temperature differentiation on inner and outer surfaces of the shell brings dramatic adjustment in results of determination of stress-strain state which is proved in works mentioned below. Articles of Treschev and Spasskaya $(2014,2015)$ consider similar problems of cylindrical orthotropic shells made of different materials with various geometrical characteristics, loads and temperatures. Works of Delyagin (2013), Treschev and Delyagin (2013) consider coupled thermo-mechanical problems of spherical shells made of isotropic different resistant graphite. The problems utilize three-dimensional finite elements. Work (Samsonenko \& Treschev, 2011) solves an uncoupled problem of bending of circular plate made of orthotropic material under quasistatic load. Works (Reddy Bert, Hsu \& Reddy, 1980; Reddy \& Bert, 1982) solve uncoupled problems of bending of orthotropic laminated plates with consideration of temperature differentiation on the surfaces of the plates within the framework of relations of Timoshenko (Timoshenko \& Woinowsky-Krieger, 1966).

In order to find temperature components in the system (12) it is necessary to consider separate problem of heat transfer through surface of the shell. The process of heat transfer is described by classic equation of heat conductivity:

$$
\frac{\partial T}{\partial t}=a_{1} \cdot T,{ }_{11}+a_{2} \cdot T,{ }_{22}+a_{3} \cdot T,{ }_{33}
$$

where $a_{1}, a_{2}, a_{3}$ - coefficients of conductivity, that characterize thermal lag of the body in corresponding directions.

The most common problems are related to stationary one-dimensional temperature field with temperature differentiation through the thickness of the structure. In this case, temperature varies depending on one coordinate (for example $\beta_{3}$ )- normal to isothermic surfaces.

In compact form for the one-dimensional case equation of conductivity will look like: $T_{{ }_{t}}=a_{3} T,_{33}$; where $t$ - current time; $a_{3}=\lambda /(\rho c)-$ coefficient of conductivity through the thickness of the shell; $\lambda$ - coefficient of conductivity; $\rho$-density of the material; $c$ - specific volumetric heating capacity of the body.

The process of linear temperature distribution through the thickness of the shell happens in a short period of time since the shell is quite thin and coefficient of conductivity is quite big. That said it is reasonable to consider the moment in time when temperature distribution stops changing.

In a specific moment in time when temperature distribution through the thickness of the shell stops changing, we can find temperature differential $\Delta T$ in any point by using linear law of heat distribution:

$$
T\left(\beta_{3}\right)=\left(T_{2}-T_{1}\right) \beta_{3} / h+\left(T_{1}+T_{2}\right) / 2-T_{0}
$$

where:

$T_{2}$ - temperature of outer surface of the shell; 
$T_{1}$ - temperature of inner surface of the shell;

$T_{0}$ - the starting temperature of the shell.

The resulting resolving differential equations is quite complex, therefore in order to solve them it is necessary to use numerical methods with method of finite differences (Varvak \& Varvak, 1977) and two-step method of consecutive parameter perturbation being in our case the easiest to realize. The resolving equations in the form of finite differences method are excessively large and therefore are not introduced in this article. The resulting system of polynomial equations are solved using Gaussian method.

\section{The solution of test problem, analysis of the results}

Let's analyze the stress-strain state of the shell, the initial scheme presented in Figure 2. Geometrical characteristics: length of the shell $L=2.0 \mathrm{~mm}$; depth of the shell $h=0.04 \mathrm{~m}$; radius of the middle surface of the shell $R=0.4 \mathrm{~m}$. Thickness to radius ratio of the shell is $\frac{h}{R}=\frac{1}{10}$. With this value solution error according to Kirchhoff-Love model does not ex- ceed 5\% in comparison with more precise theories of Timoshenko (Timoshenko \& Woinowsky-Krieger, 1966), Ambartsumian (1974), Pikul (2009).

One end of cylindrical shell is rigidly fixed, and the other is free with no load. The load is evenly distributed $q=8 \mathrm{MPa}$ and is placed perpendicularly to inner surface of the shell. Temperature mode: heat transfer happens due to heat conductivity of the shell; on the inner surface of the shell, the temperature is constant $T_{1}=0^{\circ} \mathrm{C}$; on the outer surface of the shell, the temperature is also constant $T_{2}=30^{\circ} \mathrm{C}$; the initial temperature of the shell is $T_{0}=0^{\circ} \mathrm{C}$. The material of the shell - three-reinforced woven polymer P36-50 (Roze, Zhigulin $\&$ Dushin, 1970), with the following mechanical properties: modules of elasticity $-E_{1}^{+}=10.3 \mathrm{GPa}, E_{1}^{-}=11.77 \mathrm{GPa}, E_{2}^{+}=$ $=17.6 \mathrm{GPa}, E_{2}^{-}=18.54 \mathrm{GPa}$; Poisson's ratios $-v_{12}^{+}=0.188, v_{12}^{-}=0.215$. Temperature characteristics of the material: coefficients of thermal linear expansion $\alpha_{1}^{ \pm}=33 \cdot 10^{-5}{ }^{\circ} \mathrm{C}^{-1}, \alpha_{2}^{ \pm}=40 \cdot 10^{-5}{ }^{\circ} \mathrm{C}^{-1}$ (Kargin, 1972).

Figures 3-5 show some most characteristic results. The solutions shown here utilize the model of Treschev (2016) with consideration of thermal action on the cylindrical shell.
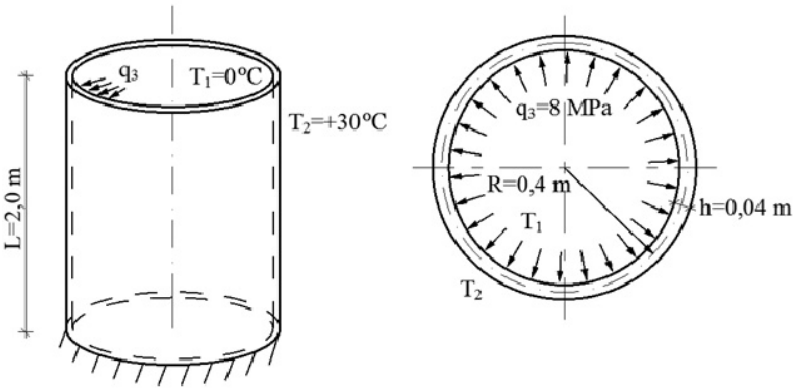

FIGURE 2. Scheme of the test problem 


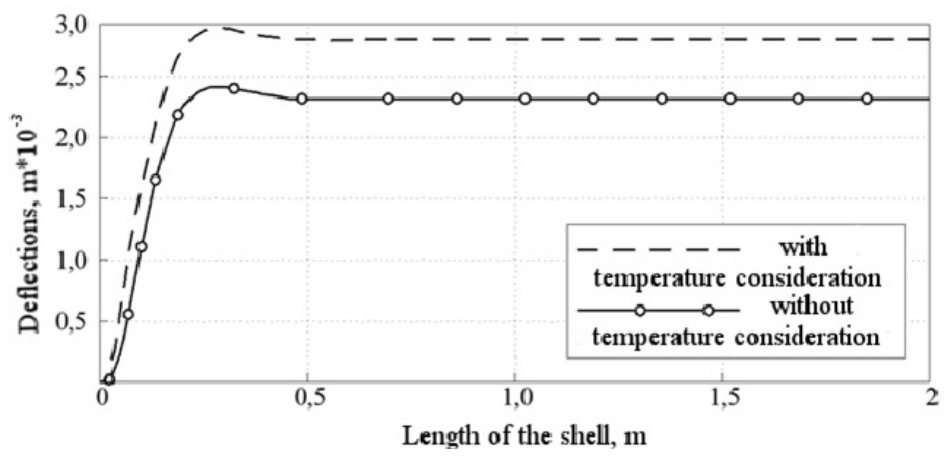

FIGURE 3. Deflections of the shell

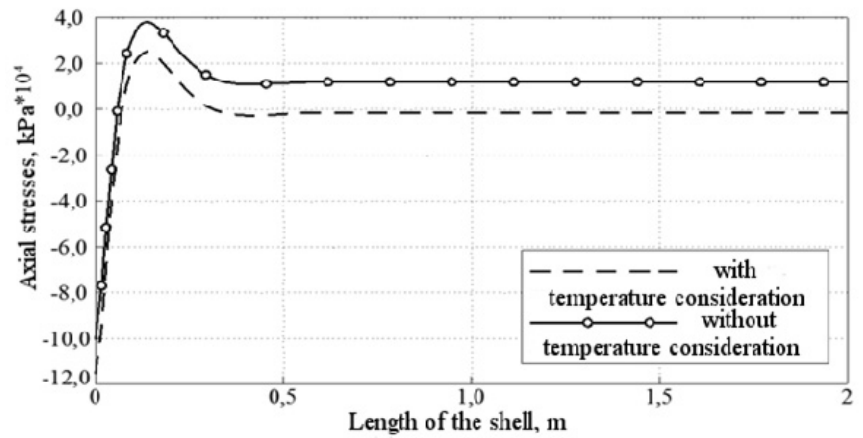

FIGURE 4. Axial stresses

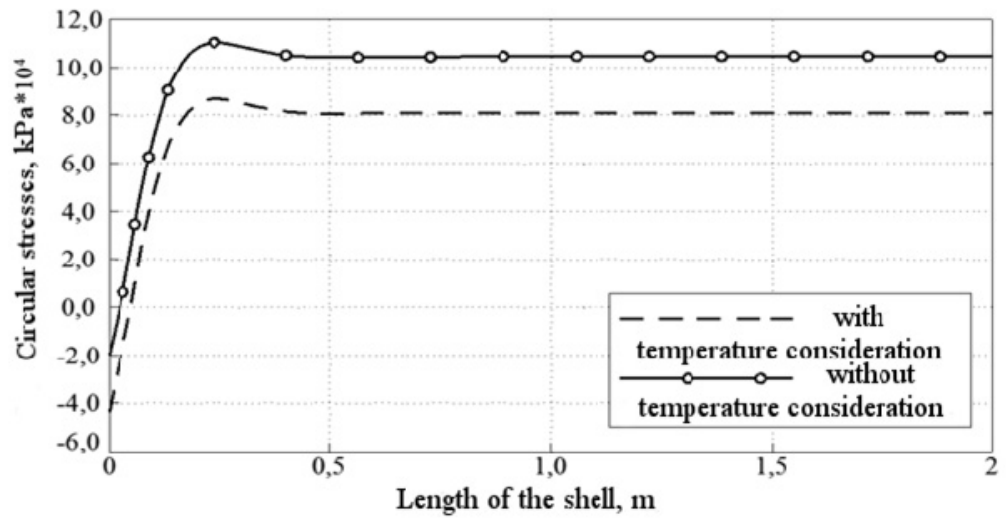

FIGURE 5. Circular stresses

Differences in maximal deflections with and without consideration of thermal action is $20.8 \%$.
For axial and circular stresses on the inner surface of the shell the numbers with and without consideration of thermal action 
are even. This is because the temperature of the inner surface of the shell is equal to the initial temperature of the shell. Therefore it is necessary to analyze the stresses on the outer surface of the shell.

For axial stresses on the outer surface of the shell numbers without consideration of thermal action are lower in the rigid part by $15 \%$ than with it. But for maximal tensile stresses numbers without the thermal action consideration exceed the contrary by $34.6 \%$. Besides at a distance of $0.125 \mathrm{~L}-\mathrm{L}$ the temperature action changes the sign of the stresses.

For circular stresses on the outer surface of the shell numbers without consideration of the thermal action in rigid part more than twice lower than with it. For maximal circular stresses on the outer surface of the shell numbers with consideration of the thermal action are lower than the contrary by $21.5 \%$.

\section{Conclusion}

The problem considers circular cylindrical orthotropic shell in force and temperature fields. It uses relations of Treschev as defining. According to research, these relations are the most accurate, when it comes to deformations of different resistant orthotropic composite materials in all types of stress-strain states.

After thorough analysis of the test problem we can conclude that consideration of the thermal action is necessary for reliable results as it greatly adjusts the stress-strain state of the structure. For example, the value of the deflections in the problem varies by more than $20 \%$ and values of stresses with and without consideration of thermal action differ more than twofold. On top of this, in order to get dependable data on stress-strain state of the shells it is necessary to consider the properties of anisotropy and different resistance using reliable theories that agree with experimental data.

\section{References}

Ambartsumian S.A. (1974). General theory of anisotropic shells. Moscow, Russia: Izdatelstvo Nauka.

Delyagin, M.U. (2013). Research of a stress-strain state of a spherical shell made of different resistant material under thermal-mechanical action using FEM. Scientific-technical Herald of Povolzhye, 4, 40-45.

Ilushin, A.A. (1963). Plasticity. Moscow, Russia: Publisher of the AS USSR.

Kargin, V.A. (1972). Encyclopedia of polymers. Moscow, Russia: Soviet encyclopedia.

Pikyl, V.V. (2009). Mechanic of shells. Vladivostok, Russia: Dalnauka.

Reddy, J.N., Bert, C.W., Hsu, Y.S., Reddy, V.C. (1980). Termal bending of thick rectangular plates of bimodulus composite materials. $\mathrm{JO}_{\mathrm{O}}$ urnal of Mechanical Science, 22(6), 297-304.

Reddy, J.N., Bert, C.W. (1982). On the Behovior of Plates Laminated of Bimodulis Composite Materials. ZAMM, 62(6), 213-219.

Roze, A.V., Zhigulin, I.G., Dushin, M.N. (1970). Three-reinforced woven materials. Mechanics of Polymers, 3, 471-476.

Samsonenko, G.I., Treschev, A.A. (2011). Thermo-elastic bending of thin circular plates made of orthotropic fiberglasses. News of TSU. Technical Sciences, 5(1-3), 116-121.

Spasskaya, M.V. (2013). Solution path to the problem of thermoelastic circular cylindrical shell made of anisotropic different resistant materials. Magistrate Herald, 7, 63-65.

Timoshenko, S.P., Woinowsky-Krieger, S. (1966). Theory of plates and shells. Moscow, Russia: Izdatelstvo Nauka.

Treschev, A.A. (2016). Theory of deformation and strength of sensitive to type of stress materials. Defining correlations. Moscow; Tula, Russia: RAACS; TSU. 
Treschev, A.A., Delyagin, M.U. (2013). Simulation of a shell made of isotropic different resistant graphite using volumetric finite elements with consideration of coupling of stresses and temperatures. Materials Physics and Mechanics, 17(1), 59-70.

Treschev, A.A., Spasskaya, M.V. (2014). The problem of thermo-elasticity for the cylindrical shell made of anisotropic different resistant material. News of TSU. Technical Sciences, 11(1-2), 567-575.

Treschev, A.A., Spasskaya, M.V. (2015). Thermo -elastic deformation of cylindrical shell made of anisotropic different resistant material. Herald of CGPU im. I.I. Iakovleva. Series: Mechanics of Limit State 1(23), 65-74.

Varvak, P.M., Varvak, L.P. (1977). Method of meshes in analysis of engineering structures. Moscow, Russia: Stroiizdat.

\section{Summary}

The influence of temperature differences for the analysis of thin orthotropic cylindrical shell. A problem of elasticity of cylindrical shell made of orthotropic different resistant material is considered in this article. The problem is uncoupled, i.e. it consists of two separate problems: of structural mechanics and of thermodynamics. The article contains research of a shell within the framework of shallow shells theory. The solution is based on the technical hypotheses of Kirchhof-Love. The relations of Treschev are used in the capacity of physical dependencies. The main relations are used in the form of the elastic solutions method of Ilushin. The process of heat transfer is described by classic equation of heat conductivity. The article introduces a system of solving equations in combined form supplemented by initial conditions and boundary conditions. The object of the problem is a shell that suffers evenly distributed load placed perpendicularly to the inner surface of the shell as well as thermal action in the form of temperature differentiation on the inner and outer surfaces of the shell. The shell is being examined in the particular moment of time when the tempe- rature transfer has subsided. The article contains the solution of the specific problem of thermo-elastic bending of thin circular cylindrical shell made of three-reinforced woven polymer. Some specific results of stress-strain state analysis are shown: deflections of the shell, axial and circular stresses, followed by the comparison of the results with and without consideration of thermal action, as well as analysis of influence of thermal action on the stress-strain state of the shell.

\section{Authors' addresses:}

Alexandr Anatolyevich Treschev, Tula State University (TSU)

Department of Engineering, Constructional

Materials and Structures

pr. Lenina 92, Tula, 300012

Russia

e-mail: taa58@yandex.ru

Maria Vladimirovna Spasskaya

Tula State University (TSU)

Department of Engineering,

Constructional Materials and Structures

pr. Lenina 92, Tula, 300012

Russia

Michael Borisovich Shereshevsky

Tula State University (TSU)

pr. Lenina 92, Tula, 300012

Russia

Alexander Anatolievich Bobryshev

Kazan (Volga region) Federal University

Department of Materials, Technologies and

Quality

Automobile Department of the Naberezhnye

Chelny Institute

Soyumbike Avenue, 84 sq. M. 68

423800, Naberezhnye Chelny

Republic of Tatarstan

Lenar Nurgalevich Shafigullin

Kazan (Volga region) Federal University

Department of Materials, Technologies and

Quality

Automobile Department of the Naberezhnye

Chelny Institute (branch)

Kol Gali boulevard, 9 sq. M. 132, Naberezhnye

Chelny

Republic of Tatarstan 